\section{Impact evaluations of mental health programmes: the missing piece in global mental health}

\author{
Mary J De Silva
}

\section{THE MISSING PIECE}

The past 10 years have witnessed a remarkable rise in the visibility of the field of global mental health, which applies the core principles of global health (improving health and equity in health for all people worldwide ${ }^{1}$ ) to mental health. The stated goal of global mental health is to reduce the burden of mental disorders using an evidence-based and human rights approach with a focus on low and middle income countries (LMIC) as this is where inequity in mental health treatment and care is the greatest. ${ }^{2}$

The field, as witnessed by the changing nature of papers published in this journal, has moved rapidly from research into the prevalence of and risk factors for mental disorders, ${ }^{3} 4$ to randomised controlled trials (RCTs) of interventions in lowresource settings. ${ }^{5}$ There is now a small but growing evidence base of RCTs of effective interventions for a range of mental disorders, largely delivered as psychological therapies by non-specialist health workers. ${ }^{6}$ This is complemented by a rich history of transcultural psychiatric research emphasising the need for locally adapted and culturally appropriate interventions, from which global mental health draws inspiration. ${ }^{7}$ The relative youth and fast-moving nature of the field means that these streams of research must continue informing each other for many years to come. For example: we lack even the most basic prevalence data from many LMIC to inform Global Burden of Disease estimates; ${ }^{8}$ interventions to combat social determinants and prevent mental disorders are poorly understood; many types of mental health interventions do not have proof of concept in lowresource settings; and we need to better understand how to adapt interventions with proof of concept to ensure they are culturally acceptable and effective in new settings.

The critical missing piece is evidence of impact from real-world mental health

Correspondence to Dr Mary J De Silva, Centre for Global Mental Health, London School of Hygiene and Tropical Medicine, Keppel Street, London WC1E 7HT, UK; mary.desilva@Ishtm.ac.uk programmes. The number of mental health programmes being run by nongovernmental organisation (NGOs), governments and public/private partnerships in LMIC dwarfs the number of RCTs that have been conducted. ${ }^{9}$ The shared learning that could be generated from these programmes is a vast and largely untapped resource that the field must draw on if we are to meet the increasing calls from policy makers for evidence of best practice to be scaled up.

\section{THE NEED FOR IMPACT EVALUATIONS IN GLOBAL MENTAL HEALTH}

A thoughtful evaluation of a real-world programme can provide vital evidence on, among other things: whether programmes work at scale; how they integrate into and affect the wider health, social, educational and justice systems; and their cost relative to other programmes. Such information is critical for policy makers to make informed decisions about which programmes to invest in. A second driver is the increasing expectation and pressure from donors results-based frameworks, which demand evidence of impact quickly and therefore force programmes to prioritise outcomes which are measurable within the time frame of the grant. Without robust methods and clear guidelines on how to measure programme impact in a meaningful way, these efforts will not deliver on their promise.

The value of impact evaluations is aptly demonstrated in other areas of global health such as malaria, HIV/AIDS and maternal and child health, whose rich and informative evidence from impact evaluations are used by donors not only to assess the effectiveness of individual programmes, but also to track progress towards international goals. ${ }^{10}$ Indeed, this new focus on outcomes of programmes in an attempt to introduce evidence-based decision-making for the allocation of resources, is seen as one of the key contributions of global health. ${ }^{11}$

Such a focus is currently absent in global mental health. A search of the 3 ie database of impact evaluations in development reveals that less than $2 \%$ of the 2500 or more evaluations are related to mental health, and none are impact evaluations of real-world mental health programmes, but are rather RCTs of specific development (such as poverty alleviation) or mental health (such as psychological therapies) interventions. ${ }^{i}$ The drive for impact evaluation within other areas of global health must be replicated within global mental health.

Isolated efforts have been made to systematically document mental health programmes, such as the case studies programme which has so far conducted detailed case studies of nine programmes. ${ }^{12} 13$ However, a key finding of this research is that almost none of the programmes conducted adequate internal or external evaluations. ${ }^{9} 12$ Lastly, in a systematic review of impact evaluations in peer-reviewed and grey literature conducted in 2013, we found only 136 evaluations of the coverage, effectiveness and/or costs of mental health programmes globally. ${ }^{14}$ Only 14 of these programmes were from LMIC.

This situation is slowly improving, as evidenced by the higher profile afforded to mental health programmes through the ongoing PLoS Medicine series on global mental health practice. ${ }^{15}$ Five of the eight papers in the series describe mental health programmes (the other three are descriptions of policy processes or health system reforms), and all five report some form of evaluation-largely an analysis of M\&E data routinely collected by the programme, with four of the five reporting patient outcomes.

\section{WHAT KIND OF IMPACT EVALUATIONS ARE NEEDED?}

A careful balance must be struck between the need to generate robust evidence, and the burden placed on the programme by the evaluation.

\section{Evaluation framework}

To strike this balance, we have recommended three aspects of programmes to be evaluated, based on the Organization for Economic Cooperation and Development (OECD) Health Care Quality Indicators (HCQI) framework. ${ }^{16}$ These comprise: quality of care including the effectiveness of the programme in improving patient outcomes; access to care measured through service utilisation and what proportion of the target population use the programme (coverage), and the cost of care including ideally the cost-

ihttp://www.3ieimpact.org/en/evidence/ impact-evaluations/ 
benefit (cost per healthy life-year gained) of the programme to enable it to be compared with other programmes. ${ }^{17}$ This complements a new initiative by the International Consortium for Health Outcomes Measurement (ICHOM) ${ }^{\mathrm{ii}}$ to develop a standard set of outcome indicators for depression and anxiety. If repeated for other mental disorders, this would provide a standard set of outcome indicators which can be used to compare the effectiveness of programmes across different settings.

It is clear that impact evaluations must be grounded in the programmes they are evaluating, address local priorities, and explore the process of implementation in order to determine how and why they do or do not work. In addition to the three impact domains outlined above, we have added a fourth category: an evaluation of the process of implementing the programme including barriers to implementation and strategies for overcoming these, in order to understand how and why a programme works in a particular context. ${ }^{17}$

Many existing impact evaluations are limited by not having an explicit theoretical framework on which to hang questions of access, process, cost and quality. Theory of Change (ToC) is a vital tool to provide a structure for the evaluation, combining process and outcome evaluations into a single theoretical framework, ${ }^{18}$ with the added benefit of ensuring that evaluations address local priorities and have the buy in of local stakeholders. ${ }^{19}$ ToC is a theory, visually represented as a causal pathways map, of how and why a programme works. This theory is empirically tested by measuring indicators for every expected outcome on the hypothesised causal pathway to impact. It is developed in collaboration with stakeholders and modified throughout the intervention development and evaluation. Colleagues and I are using ToC in a range of mental health programmes including RCTs and non-randomised evaluations of mental health programmes, and recommend its use to provide a theoretical framework for impact evaluations (E Breuer. The use of Theory of Change to design, implement and evaluate public health interventions: a systematic review, personal communication, 2014). ${ }^{18}$

\section{Research designs}

The research designs used for impact evaluations must strike a balance between scientific rigour and feasibility. Pragmatic

ii http://www.ichom.org/

RCTs in which the intervention is delivered in the same circumstances were it to be rolled out are the gold standard, but are frequently not possible when adequate funding and the appetite of key stakeholders for a control group are not present. This is particularly true in global mental health where limited donor funding exits for research and programme implementation, and where large units of randomisation (such as local government authority areas) are often needed to preserve the fidelity of the intervention, making the trial more costly and logistically difficult. More realistic is the careful selection of a comparison group of health facilities or districts where the programme is not being implemented, though this in itself requires significant additional research resources to collect data in the control arm, and can lead to significant bias if the selected control areas are systematically different from the intervention areas. As a minimum, independent research evaluation of pilot projects implementing new programmes should be considered the norm, comprising a cohort of patients treated by the programme to evaluate changes in clinical, functioning, and economic outcomes. However, with funding so scare for impact evaluations in general and global mental health specifically, this may also be an unrealistic expectation.

The most practical solution is a tool kit with which programmes can conduct their own monitoring and evaluation, tracking the process of implementation, costs, and ultimately changes in outcomes that matter to service users and families over time through a robust Mental Health Information Systems (MHIS). Colleagues and I have started some of this work with the international NGO CBM International in Nigeria, working with them to develop simple routine data collection forms which form part of the patients' clinical notes and which are completed by programme staff at key contact points with service users (enrolment in the programme, every 6 months and at discharge). These data must feed into regional and national level Health Management Information Systems (HMIS) to incentivise staff to collect robust and valid data on mental health.

\section{Challenges}

Impact evaluation in low-resource settings is not easy. Significant barriers include the lack of routinely reported data on mental health so that often no data on mental health diagnoses, treatment or outcomes are available in the HMIS. Collecting routine data on mental health is particularly problematic in LMIC where underdiagnosis of most mental health problems is the norm. ${ }^{20}$ Without the development of robust MHIS which feed directly into national level HMIS, and capacity building and ongoing support for the staff who collect these data, the amount of evidence generated by impact evaluations of mental health programmes, and the ability the track the impact of initiatives to reduce the treatment gap more broadly, will remain pitifully small.

Challenges to conducting independent impact evaluations include the development of new methods to measure access, process, cost and quality in a simple and cost effective way, and the selection of locally valid tools to assess mental health outcomes in diverse cultural contexts. Complete impact evaluations covering the four domains of access, process, cost and quality are complex as they measure multiple programmatic aspects at different levels of the health system, include the impact on other sectors including social and educational, and involve a range of data collection methods requiring multidisciplinary expertise. ${ }^{17}$ However, the rewards of such an evaluation are great.

\section{THE WAY FORWARD}

Three things need to happen for impact evaluations to take their rightful place in global mental health. First, comprehensive evaluations of mental health programmes delivered at scale need to happen. One significant example is the Department for International Development (DFID) funded PRogramme for Improving Mental health carE (PRIME) which is developing and evaluating district level mental healthcare plans in five countries and evaluating them at the district level. ${ }^{21}$ The independent research evaluation is very comprehensive, including changes in coverage, patient outcomes, costs and the process of implementation across all five districts, using a ToC framework. $^{22}$ More funding for similar evaluations is needed, with existing funding for impact evaluations (such as that from DFID and 3ie) made available to mental health programmes.

Second, we need to develop robust, easy to implement and cheap methods of evaluating mental health programmes at scale, ideally using a common evaluation framework that can be applied across programmes and used to compare the relative impact of different programmes, such as through the ICHOM programme. The results of these evaluations should be modelled using tools such as OneHealth ${ }^{\mathrm{iii}}$

iiihttp://www.futuresinstitute.org/onehealth.aspx 
to predict the financial and human resource costs of scaling up particular programmes relative to other programmes in a given setting, to provide robust evidence for policy makers. Such evidence can contribute to global efforts to evaluate the potential costs and benefits of allocating funding to different health programmes, such as the forthcoming Disease Control Priorities 3rd edition which includes a chapter on mental disorders. ${ }^{\text {iv }}$

Third, we need a mechanism to share the results of these evaluations so that new programmes can learn from the lessons of the ones that have gone before, and to engage policy makers with the evidence base. An exciting opportunity exists in the launch of the Mental Health Innovation Network (MHIN), a largely internet-based network of mental health researchers, practitioners and policy makers funded by Grand Challenges Canada. ${ }^{\mathrm{v}} \mathrm{MHIN}$ hosts a rapidly growing database of over 90 case studies of mental health programmes from around the world. Profiling programmes in this way provides easily accessible evidence of good practice for researchers seeking to adapt existing programmes to new contexts, and for policy makers and donors seeking to invest in scaling up best practice. In addition, MHIN is able to develop and host capacity building materials for programme evaluation, including a tool kit for MHIS and independent impact evaluations. Evidence collated in this way can be quickly accessed by policy makers to advocate for more and better mental health services, such as at the World Innovation Summit for Health $^{23}$ and at the World Bank meeting on depression in Autumn 2015.

\section{CONCLUSION}

Impact evaluations are not the panacea for global mental health - but they do represent an important evolution and maturation of the field and are critical if we are to influence donors and governments to invest more heavily in mental health. Until robust evidence on the impact, costs and process of mental health programmes is more widely available, efforts to scale up evidence-based services will be hampered.

The ultimate goal of impact evaluation in global mental health must be to conduct independent evaluations of much larger programmes delivered at scale using

\footnotetext{
${ }^{\text {iv } h t t p: / / w w w . d c p-3 . o r g / n o d e / 108 ~}$

${ }^{v}$ www.mhinnovation.net
}

routinely collected data, such as has been done in maternal and child health to track progress towards the Millennium Development Goals. ${ }^{24}$ We are perhaps years away from this, as global goals for mental health do not yet exist, routine data on mental health is almost never collected in LMIC, and mental health outcomes are not as clear cut as maternal mortality. However, such evaluations must be the ultimate goal if we are to generate international interest in tracking progress towards, and ultimately achieving, a reduction in the treatment gap for mental disorders in low-resource settings.

Acknowledgements The author is grateful to Vikram Patel and Alex Cohen for providing comments on an earlier version of this commentary. With thanks to members of the Mental Health Innovation Network Team who constructed the innovation database: Lucy Lee, Grace Ryan, Marguerite Regan, Shamaila Usmani, and Gemma-Claire Ali, and to Grace Ryan, Julian Eaton and Alex Cohen for developing the M\&E tools in Nigeria as part of the case studies project. This commentary is an output of the PRogramme for Improving Mental health carE (PRIME), funded by UKAID from UK Government.

Funding MJDS is funded by a Wellcome Trust/LSHTM fellowship.

Competing interests None.

Provenance and peer review Commissioned; externally peer reviewed.
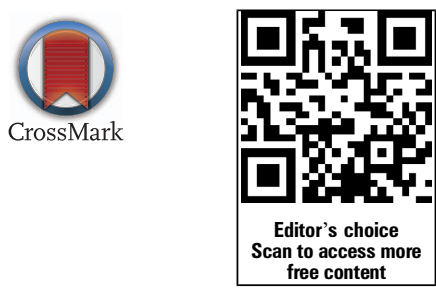

To cite De Silva MJ. J Epidemiol Community Health 2015;69:405-407.

Published Online First 9 December 2014

J Epidemiol Community Health 2015;69:405-407. doi:10.1136/jech-2014-203866

\section{REFERENCES}

1 Koplan J, Bond T, Merson M, et al. Towards a common definition of global health. Lancet 2009;73:1993-5.

2 Patel V, Prince M. Global mental health. A new global health field comes of age. JAMA 2010;303:1976-7.

3 Araya R, Lewis G, Rojas G, et al. Education and income: which is more important for mental health? J Epidemiol Community Health 2003;57:501-5.

4 Amone-P'Olak K, Stochl J, Ovuga E, et al. Psychological wellbeing and mental health: postwar environment and long-term mental health problems in former child soldiers in Northern Uganda: the WAYS study. J Epidemiol Community Health 2014;68:425-30

5 Han C, Ssewamala F, Wang J. Family economic empowerment and mental health among
AIDS-affected children living in AIDS-impacted communities: evidence from a randomised evaluation in southwestern Uganda. J Epidemiol Community Health 2013:67:225-30.

6 van Ginneken N, Tharyan P, Lewin S, et al. Non-specialist health worker interventions for the care of mental, neurological and substance-abuse disorders in low and middle-income countries. Cochrane Database Syst Rev 2013;11:CD009149.

7 Kirmayer L, Swartz L. Culture and mental health. In: Patel V, Minas H, Cohen A, et al., eds. Global mental health: principles and practice. Oxford: Oxford University Press, 2014

8 Baxter A, Patton G, Scott K, et al. Global epidemiology of mental disorders: what are we missing? PLOS ONE 2013;8:e65514.

9 Eaton J, McCay L, Semrau M, et al. Scale up of services for mental health in low-income and middle-income countries. Lancet 2011:378. 1592-603.

10 Graham W, Filippi V, Ronsmans C. Demonstrating programme impact on maternal mortality. Health Policy Plan 1996;11:16-20.

11 Liden J. The grand decade for global health: 19982008. London: Chatham House (The Royal Institute of International Affairs), 2013.

12 Cohen A, Eaton J, Radtke B, et al. Three models of community mental health services in low-income countries. Int J Ment Health Syst 2011;5:3.

13 Cohen A, Raja S, Underhill C, et al. Sitting with others: mental health self-help groups in northern Ghana. Int J Ment Health Syst 2012;6:1.

14 De Silva $M$, Lee $L$, Fuhr $D$, et al. Estimating the coverage of mental health programmes: a systematic review. Int J Epidemiol 2014:43:341-53.

15 Patel V, Jenkins $R$, Lund $C$, et al. Putting evidence into practice: the PLoS medicine series on global mental health practice. PLoS Med 2012;9:e1001226.

16 Kelley E, Hurst J. Health Care Quality Indicators Project: Conceptual framework paper. OCED Health Working Papers No 23: OCED, 2006.

17 De Silva M, Cohen A, Patel V. Evaluation of interventions in the real world. In: Thornicroft $G$, Patel V, eds. Global mental health trials. Oxford: Oxford University Press, 2014

18 De Silva M, Breuer $E_{\text {, Lee }} \mathrm{L}$, et al. Theory of change: a theory-driven approach to enhance the MRC framework for complex interventions. Trials 2014:15:267.

19 Anderson A. The community builder's approach to Theory of Change. A practical guide to theory development. New York: The Aspen Institute, 2005.

20 Kauye F, Jenkins $R$, Rahman A. Training primary health care workers in mental health and its impact on diagnoses of common mental disorders in primary care of a developing country, Malawi: a cluster-randomized controlled trial. Psychol Med 2013;44:657-66.

21 Lund C, Tomlinson M, De Silva MJ, et al. PRIME: a Programme to reduce the treatment gap for mental disorders in five low- and middle-income countries. PLoS Med 2012;9:e1001359.

22 De Silva M, Rathod S, Hanlon C, et al. Methods to evaluate district mental health care plans in 5 low and middle income countries, In preparation.

23 Patel V, Saxena S, De Silva M, et al. Transforming lives, enhancing communities: innovations in mental health. World Innovation Summit for Health. Qatar: Qatar Foundation, 2013.

24 Lozano R, Wang $\mathrm{H}$, Foreman $\mathrm{K}$, et al. Progress towards Millennium Development Goals 4 and 5 on maternal and child mortality: an updated systematic analysis. Lancet 2011;378:1139-65. 